\title{
A tale of two SCADs: Spontaneous coronary artery dissection series
}

\author{
Sanjana Rao BS, Nitish Mittal BS, Mohammad M Ansari MD
}

\begin{abstract}
Spontaneous coronary artery dissection (SCAD), a relatively rare condition, occurs when a tear develops in the coronary artery wall that may slow blood flow and lead to clot formation. This occurs mostly in relatively young and healthy women with minimal past medical histories; most patients have chest pain or shortness of breath. In this report, we present two female SCAD patients and discuss the etiology, diagnosis, and treatment of SCAD. We describe a 33-year-old woman and a 40-year-old woman, both presenting to the emergency department with chest pain and shortness of breath. Coronary artery angiography demonstrated SCAD. Both patients were treated with aspirin, beta-blockers, and statins with good clinical responses; one patient required a coronary stent.
\end{abstract}

Keywords: Spontaneous coronary artery dissection, female, intramural hematoma

\section{INTRODUCTION}

Spontaneous coronary artery dissection (SCAD) is a spontaneous tear in the wall of a coronary artery that often occurs in young, healthy female patients (Figure 1). The reasons for spontaneous tears include postpartum stress, heavy exertion when exercising, connective tissue disorders, and certain medications. Female patients presenting with symptoms, such as chest pain and shortness of breath consistent with a possible heart attack but no history of atherosclerosis, may need evaluation for SCAD, especially if the patient has minimal past medical history or recent childbirth.

\section{CASES}

Patient 1: A woman, age 33, presented to the emergency department complaining of chest pain and shortness of breath with pain radiating into both arms and legs and numbness in both hands. The patient

Corresponding author: Mac Ansari

Contact Information: Mac.Ansari@ttuhsc.edu

DOI: $10.12746 /$ swrccc.v9i37.785 has a history of asthma and is a regular tobacco user, smoking several cigarettes per day.

Physical examination was unremarkable. Systolic and diastolic blood pressures were both elevated with a normal heart rate. Other vital signs were within the normal range. An electrocardiogram showed ST segment elevation, suggesting myocardial infarction.

Coronary artery angiography demonstrated a variation in lumen size most notable in the mid-todistal segment of the left anterior descending coronary artery, consistent with a spiral dissection (Figure 2). A left ventriculogram demonstrated severe apical hypokinesis and distal inferior akinesis with normal function present in the remaining cardiac walls (Figure 3 ).

Patient 2: A woman, age 40, presented to the emergency department with the acute onset of chest pain. The patient had undergone a recent breast reduction but otherwise had no risk factors or significant past medical history. Physical examination was unremarkable. Systolic and diastolic blood pressures were elevated.

A left anterior descending lesion completely occluded the mid segment of the vessel. A thrombotic occlusion was suspected and partial aspiration of the thrombus was successful. Since the vessel could not 

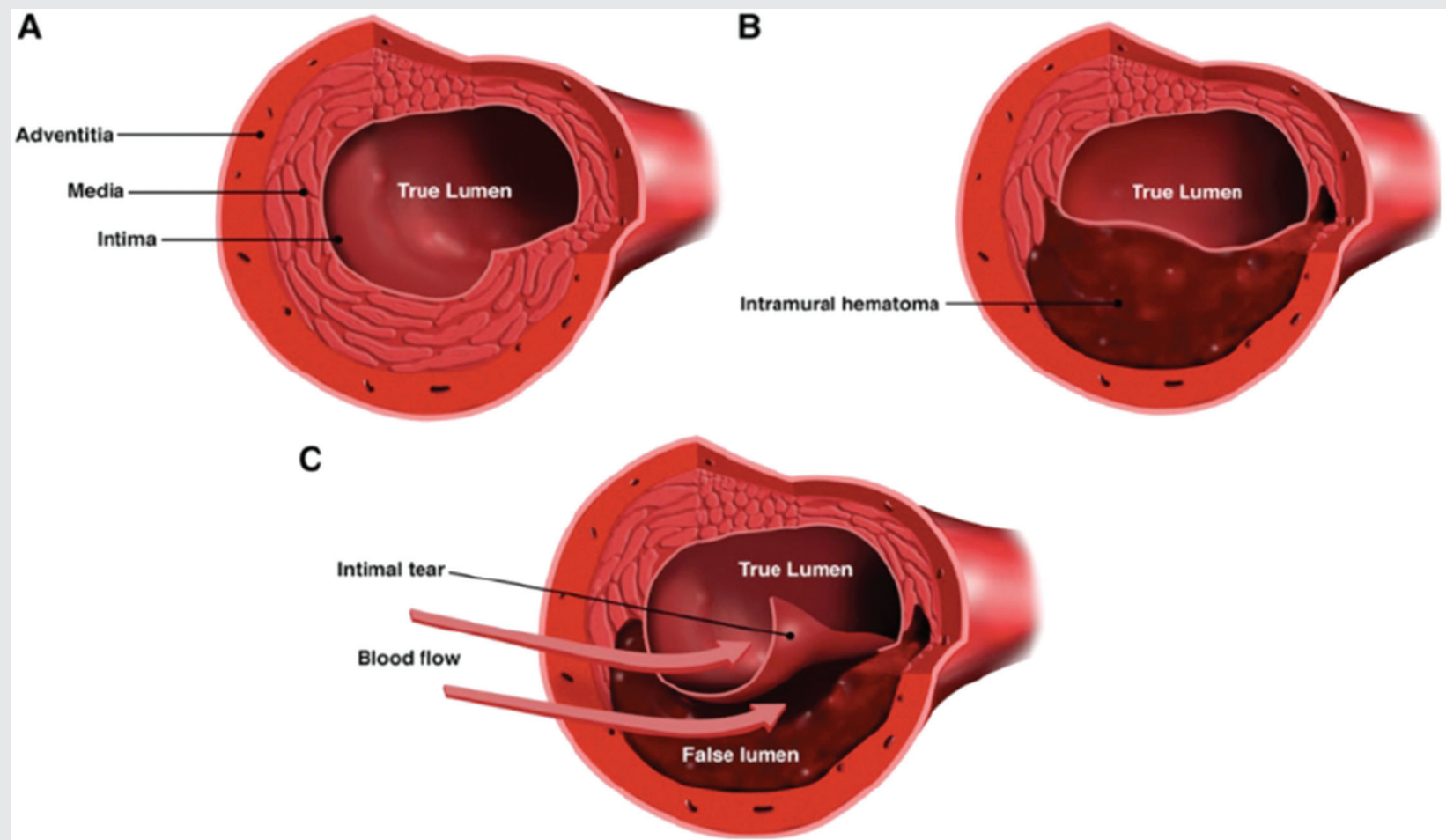

Figure 1. Spontaneous Coronary Artery Disease Blood Vessel. Vessel lumen changes present in SCAD. A. Normal blood vessel. B. Induction of vascular damage. C. True and false lumen due to an arterial dissection. ${ }^{4}$

be completely cleared of thrombus, a stent was placed in the LAD. Evidence of a flap was discovered in the distal segment suggesting that this patient had a SCAD, causing a thrombus and acute myocardial infarction. An immediate post-intervention echocardiogram demonstrated moderate apical hypokinesis of the left ventricle.

\section{Discussion}

Spontaneous coronary artery dissection is a rare condition resulting in a tear in the wall of the coronary artery, often in a specific subset of patients. The first spontaneous coronary artery dissection patient was reported in 1931 in a 42-year-old woman. ${ }^{1}$ Spontaneous dissection has since been reported in many patients, specifically in women between the ages of 18 and 84 (mean age: 44) with minimal pre-existing medical disorders. ${ }^{2} \mathrm{~A}$ dissection is found in $0.07-0.20 \%$ in all angiographies. ${ }^{2}$ The pathology of SCAD is mainly due to a false lumen developing in the outer layer of the tunica media (Figure 1). Two main theories, the 'inside-out' and 'outside-in' theories, possibly explain the development of SCAD. The inside-out model is due to the development of a tear, leading to the accumulation of blood in the media, while the outside-in model is potentially due to a vasa vasorum microvessel disruption, leading to hemorrhage. ${ }^{2}$

Specific causes of SCAD can be grouped into three categories: atherosclerosis, pregnancy-related, and other causes. Pregnancy has been shown to increase the risk for SCAD with an incidence of 1.8 SCAD cases per 100,000 pregnancies in a study with data collected between 2008 and 2010. ${ }^{2}$ Pregnancy and postpartum SCAD may have more complications with higher percentages of ST segment elevation myocardial infarction, cardiac arrest, and death. ${ }^{2}$ The physiology for SCAD's association with pregnancy has been attributed to the hormonal changes observed during pregnancy leading to abnormal collagen synthesis. ${ }^{5}$ Other risk factors reported include atherosclerosis, mechanical stressors and exercise, emotional stressors, and certain inherited connective tissue disorders. ${ }^{2}$

The presentation of SCAD has a wide range of presentations, including shortness of breath, chest 


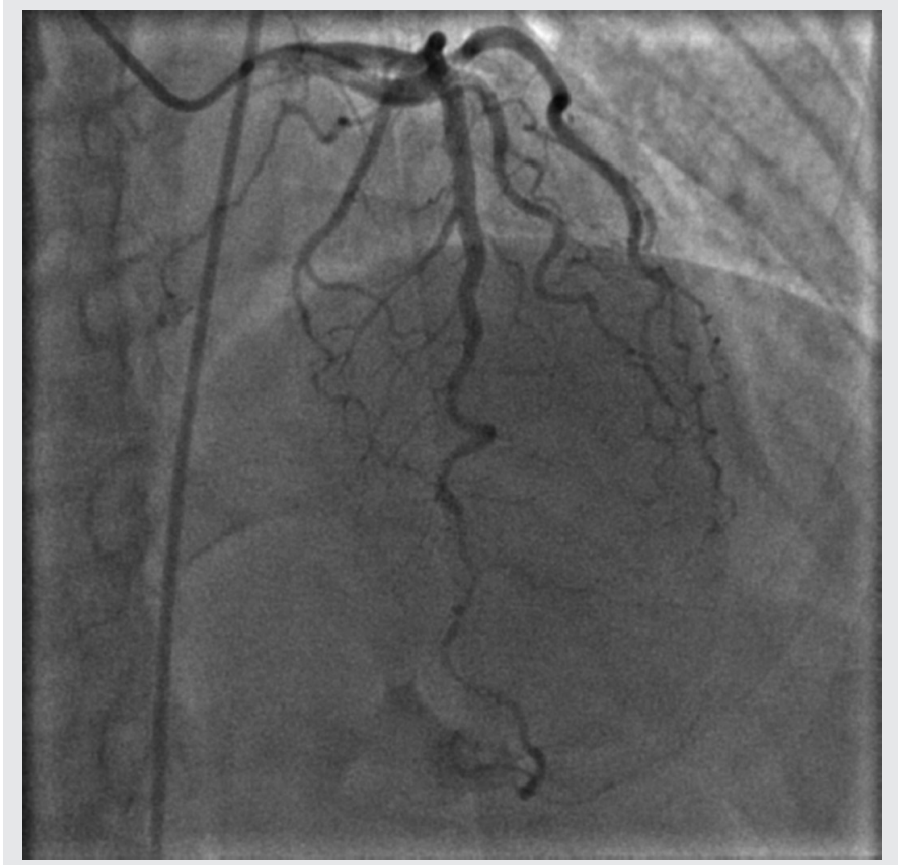

Figure 2. SCAD Coronary Artery Angiogram. Coronary artery angiogram [CRA 35] demonstrating arterial dissection for Patient 1 .

pain, and pain radiation down the upper limbs or back with varying severity. These presentations can range from a mild presentation to a severe presentation which can lead to acute heart failure. Our patients presented with similar symptoms and were evaluated for SCAD based on their risk factors and presentations. Coronary artery angiography was done to assess the potential location of the lesion or tear.

Management and treatment of SCAD depends on the severity of the dissection and the specific presentation of symptoms. Patients are usually started on aspirin, anticoagulants, cholesterol drugs, and medication to control chest pain depending on the symptom severity and the possibility of reoccurrence. ${ }^{3}$ Patients with SCAD with more severe dissections are treated with surgery or stent placement if necessary. Different treatment was used in our two patients. Patient 1 did not require stenting due to the mild nature of the dissection and was treated with aspirin, a beta blocker, and a statin medication with follow-up. However, patient 2 was stented to prevent further dissection and ensure adequate blood flow due to the presence of a residual thrombus.

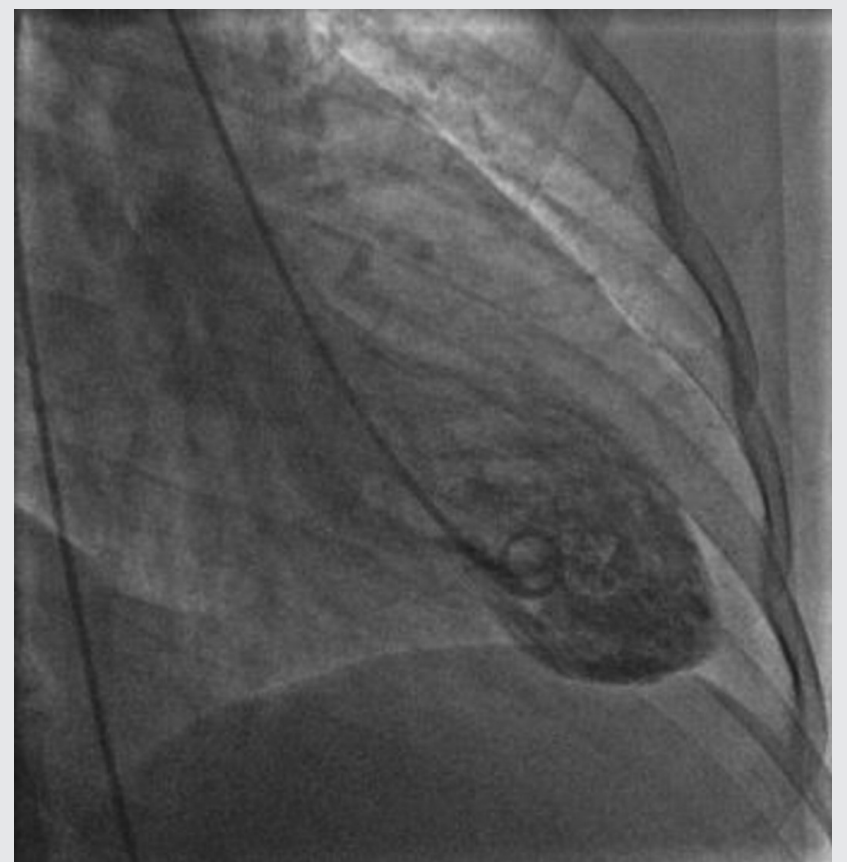

Figure 3. Left ventriculogram. Left ventriculogram [RAO 30] exhibiting functionality in the cardiac walls for Patient 1.

\section{Conclusion}

Spontaneous coronary artery dissection commonly occurs in young women with minimal risk factors. Coronary artery angiography should be used as a diagnostic tool if symptoms raise suspicion for SCAD. Treatment varies and includes medications, such as aspirin and beta blockers, for milder cases and stenting/ surgery for more severe cases.

Article citation: Rao S, Mittal N, Ansari MM. A tale of two SCADs: spontaneous coronary artery dissection series. The Southwest Respiratory and Critical Care Chronicles 2021;9(37):70-73

From: Department of Internal Medicine, Texas Tech University Health Sciences Center, Lubbock, Texas

Submitted: $12 / 16 / 2021$

Accepted: $1 / 8 / 2021$

Reviewer: Deephak Swaminath MD

Conflicts of interest: none

This work is licensed under a Creative Commons Attribution-ShareAlike 4.0 International License. 


\section{REFERENCES}

1. Mokhberi V, Bagheri B, Navidi S, et al. Spontaneous coronary artery dissection: a case report. J Tehran Heart Cent. 2015; 10(3):159-162.

2. Adlam D, Alfonso F, Maas A, et al. Writing Committee. European Society of Cardiology, acute cardiovascular care association, SCAD study group: a position paper on spontaneous coronary artery dissection. Eur Heart J. 2018;39(36):3353-3368.

3. Mayo Clinic. Spontaneous Coronary Artery Disease. https:// www.mayoclinic.org/diseases-conditions/spontaneous-coronaryartery-dissection/diagnosis-treatment/drc-20353716. Accessed 14 Dec 2020.
4. Hayes SN, Kim ESH, Saw J, et al. American Heart Association Council on Peripheral Vascular Disease; Council on Clinical Cardiology; Council on Cardiovascular and Stroke Nursing; Council on Genomic and Precision Medicine; and Stroke Council. Spontaneous Coronary Artery Dissection: Current State of the Science: A Scientific Statement from the American Heart Association. Circulation. 2018 May 8;137(19): e523-e557.

5. Yip A, Saw J. Spontaneous coronary artery dissection: A review. Cardiovasc Diagn Ther 2015;5(1):37-48. 